\title{
Successful Surgical Removal of a Huge Hepatocellular Carcinoma
}

\author{
Wang Gu, Guoqing Long, Qili Hu and Zhong Tong \\ Department of Surgery, Anhui Medical University Third Affiliated Hospital, Hefei City, Anhui Province, China
}

\begin{abstract}
Hepatocellular carcinoma (HCC) is the most common primary liver cancer. Hepatectomy and transcatheter arterial chemoembolisation (TACE) are generally accepted methods for the treatment of huge HCCs, but the most appropriate treatment is still controversial. We report a 43-year woman with giant HCC, who successfully underwent surgery. The patient was admitted with persistent right upper abdominal pain and fever. Before operation, we assessed the residual liver function and reserve capacity of the patient, and excluded the relevant surgical contra-indications. The results showed that the patient could tolerate surgical treatment, so we performed HCC resection, and the patient was successfully treated. Individualised treatment should be carried out according to tumor factors, baseline liver function and patient's functional status, so as to maximise the benefit to patients.
\end{abstract}

Key Words: Huge hepatocellular carcinoma, Hepatectomy, TACE.

How to cite this article: Gu W, Long G, Hu Q, Tong Z. Successful Surgical Removal of a Huge Hepatocellular Carcinoma. J Coll Physicians Surg Pak 2022; 32(01):105-107.

\section{INTRODUCTION}

Hepatocellular carcinoma (HCC) is the most common primary liver cancer. It is the sixth most common malignant tumor in the world and the third most common cause of cancer-related death. Studies have shown that the incidence of HCC seems to be increasing in most countries. ${ }^{1-3}$ Although significant progress has been made in the treatment of HCC, there are still major challenges in the treatment of huge HCCs (defined as $>10 \mathrm{~cm}$ ). ${ }^{4}$ Hepatectomy and transcatheter arterial chemoembolisation (TACE) are generally accepted methods for the treatment of huge HCC, but the most appropriate treatment is still controversial. It has been reported that the effect of surgical treatment is much better than that of non-surgical treatment. Precise preoperative evaluation is an important component of perioperative period and a prerequisite to ensure the feasibility of surgical resection. Preoperative residual liver function and reserve capacity can be assessed by Child-Pugh score of liver function, indocyanine green retention rate at $15 \mathrm{~min}$ (ICG-R15), and residual liver volume estimation. ${ }^{5-7}$

Correspondence to: Zhong Tong, Anhui Medical University Third Affiliated Hospital, Hefei City, Anhui Province, China

E-mail: tougao2425@163.com

Received: October 25, 2020; Revised: December 22, 2020;

Accepted: December 24, 2020

DOI: https://doi.org/10.29271/jcpsp.2022.01.105

\section{CASE REPORT}

A 43-year woman with persistent mid-upper abdominal pain was admitted to hospital. After admission, computed tomography $(\mathrm{CT})$, b-mode ultrasound and related laboratory examinations were performed. The CT scan showed a large space-occupying lesion in the right liver (Figures 1 and 2), with a lesion size of about $8 \times 15 \mathrm{~cm}$. Serum alpha-fetoprotein (AFP) was $1210 \mathrm{ng} / \mathrm{ml}$. The patient was diagnosed with giant liver cancer.

Hepatectomy is still the most effective method for the treatment of huge liver cancer, but it is necessary to fully evaluate the liver reserve function before deciding to treat the patients, so as to reduce the occurrence of postoperative liver failure. Indocyanine green (ICG) clearance test has been proved by many investigators to be safe, sensitive and accurate in quantitative assessment of liver reserve function before hepatectomy, which is helpful for early prediction and diagnosis of liver failure after hepatectomy, and has been widely used in clinic. ${ }^{7}$ Preoperative examination of patient indicated that adequate liver reserve was available, thus we decided to perform hepatectomy.

We removed the entire right liver and found that the lesion invaded the diaphragm and was adherent to the diaphragm. The resected lesion is shown in Figure 3. Involved part of diaphragm was also removed. After successful implementation of the operation and perfect nursing thereafter, the patient was eventually discharged smoothly. He was advised to follow-up in oncology clinic for further management. 


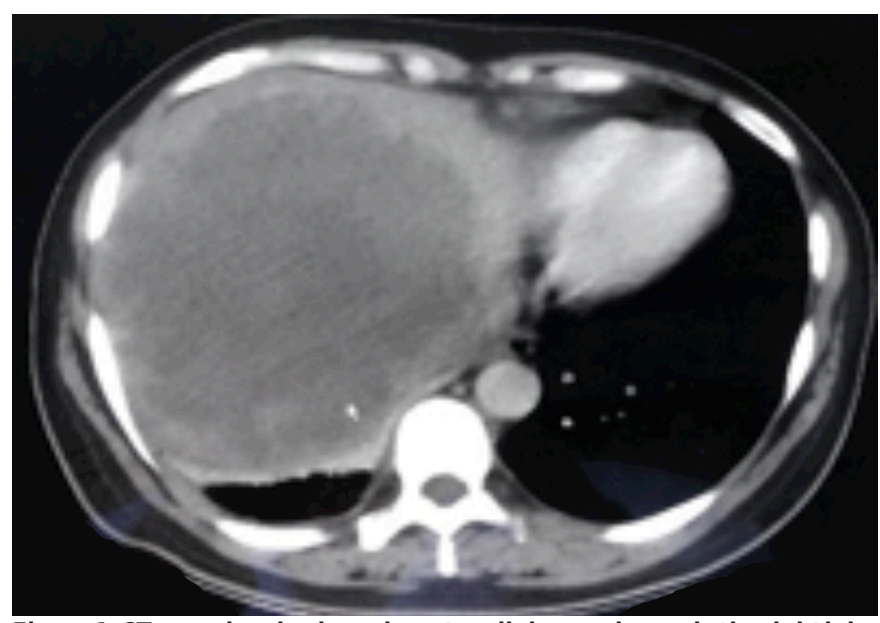

Figure 1: CT scan showing large hepatocellular carcinoma in the right lobe of liver.

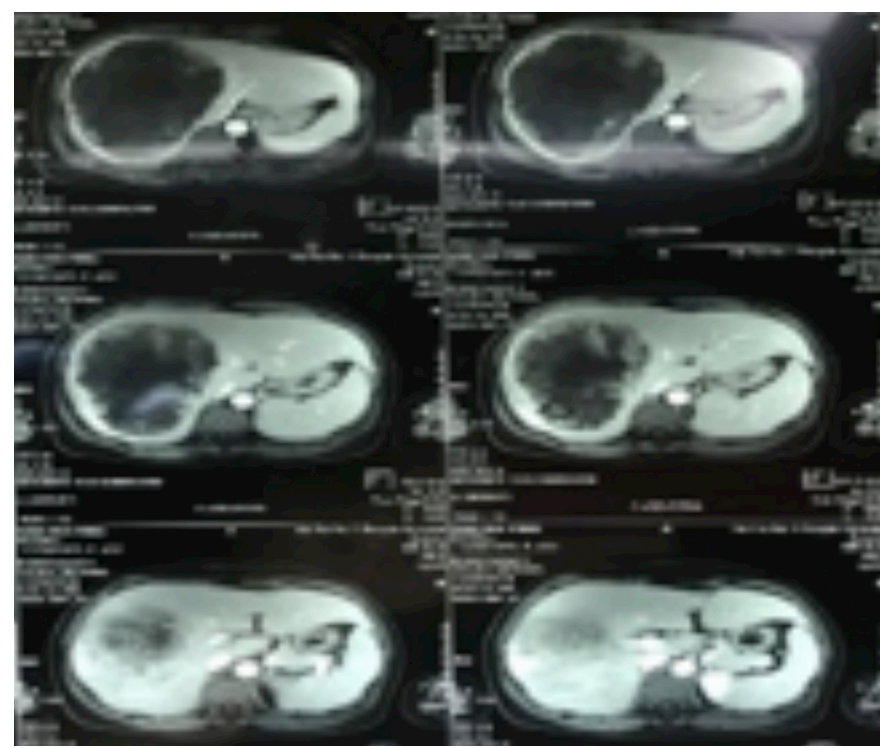

Figure 2: CT images showing the extent of hepatocellular carcinoma.

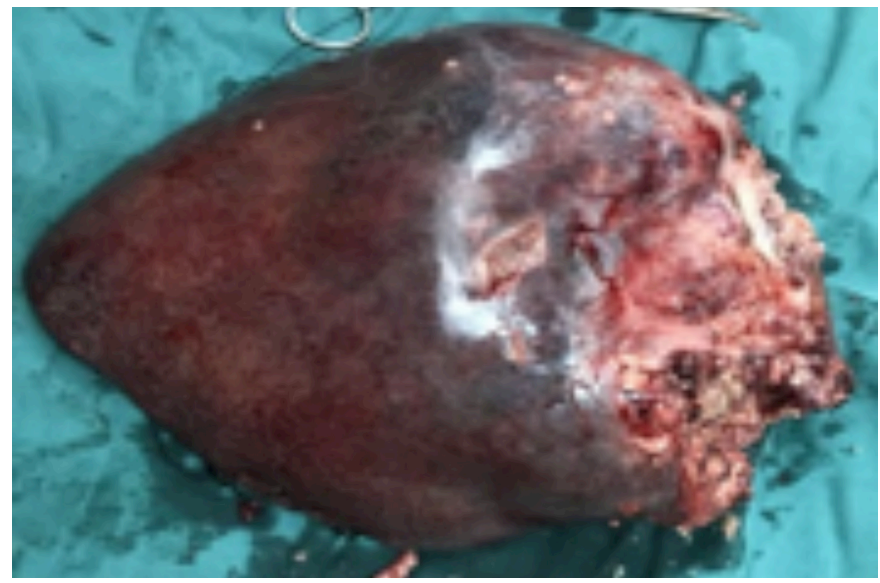

Figure 3: Gross tumorspecimen.

\section{DISCUSSION}

$\mathrm{HCC}$ is one of the most common cancers in the world. With the development of comprehensive treatment, including local and systemic treatment, HCC is no more a lethal cancer. Surgical excision is considered to be the most effective treatment. ${ }^{8}$ The choice of treatment for giant liver cancer has been controversial, but most studies have shown that hepatectomy appears to be more effective than other treatments. Firstly, huge HCC is an absolute contra-indication of liver transplantation. Secondly, radiofrequency ablation (RFA) and TACE have proved to be ineffective in the treatment of giant HCC. In addition, sorafenib, as a targeted drug, cannot achieve tumor regression. Hence, surgical resection may be the only option for giant HCC. ${ }^{9}$

Although in the past 20 years, with the advancement of surgical techniques, more and more candidates of hepatectomy schemes have been proposed for hepatectomy, there is no supplementary scheme for HCC after hepatectomy. Recent advances in local therapy, such as repetitive hepatectomy, RFA and TACE, have improved the control of intrahepatic recurrence and prognosis. However, extrahepatic metastasis has been difficult to control. Although surgical resection can remove lung or lymph node metastases, or radiotherapy is reported to be effective in some cases, systemic chemotherapy is used in most cases of unresectable or multiple metastases. Sorafenib is the only drug for unresectable HCCs; however, it is difficult for the tumors to regress completely, especially in patients with lung metastases. Studies have shown that tumors larger than $10 \mathrm{~cm}$ in diameter may be an independent risk factor for extra-hepatic recurrence, ${ }^{10}$ leading to early recurrence and poor prognosis. These results suggest that large HCC patients should be considered to be at high risk of systemic recurrence after surgery. Therefore, the use of sorafenib and other drugs for systemic adjuvant therapy is needed in the future.

Accurate preoperative assessment of liver reserve function is of great significance for the selection of reasonable treatment and prediction of postoperative recovery. Postoperative liver function recovery is mainly affected by preoperative liver function and residual liver volume. Preoperative accurate understanding of liver volume and prediction of residual liver volume can predict the recovery of liver function after surgery. Remaining liver volume or segment can be accurately measured by $\mathrm{CT}$, so the risk of operation can be well estimated. These will help surgeons choose the best treatment.

In summary, current studies have shown that hepatectomy may provide a better long-term overall survival than TACE for large single HCC without increasing mortality or morbidity. However, for large HCCs, the risks and benefits of various treatments must also be considered. Individualised treatment should be carried out, according to tumor factors, baseline liver function and patient's functional status, so as to maximise the benefit to patients.

\section{ACKNOWLEDGEMENTS:}

We would like to express our sincere gratitude to the relatives of the patient, and the staff of the hepatobiliary surgery ward.

\section{PATIENT'S CONSENT:}

Written informed consent was obtained from the patient's nextof-kin for publication of this case report and any accompanying images. 


\section{CONFLICT OF INTEREST:}

The authors declared no conflict of interest.

\section{AUTHORS' CONTRIBUTION:}

WG, GL, QH, ZT: Involved in the management.

WG: Wrote the manuscript.

ZT: Provided expert advice.

All authors read and approved the final version.

\section{REFERENCES}

1. Forner A, Reig M, Bruix J. Hepatocellular carcinoma. Lancet 2018; 391(10127):1301-14. doi: 10.1016/S0140-6736(18) 30010-2.

2. Venook AP, PapandreouC, , Furuse J, de Guevara LL. The incidence and epidemiology of hepatocellular carcinoma: A global and regional perspective. Oncologist 2010; 15(4):5-13. doi: 10.1634/theoncologist.2010-S4-05.

3. Peter F, Michael F, Douglas L. World gastroenterology organisation guideline. Hepatocellular carcinoma (HCC): A global perspective. J Gastrointestin Liver Dis 2010; 19(3): 311-7.

4. Tsoulfas G, Mekras A, Agorastou P, Kiskinis D. Surgical treatment for large hepatocellular carcinoma: Does size matter? ANZ J Surg 2012; 82(7-8):510-7. doi: 10.1111/j. 1445-2197.2012.06079.x.

5. Memeo R, de'Angelis N, de Blasi V, Cherkaoui Z, Brunetti
O, Longo $\mathrm{V}$, et al. Innovative surgical approaches for hepatocellular carcinoma. World J Hepatol 2016; 8(13): 591-6. doi: 10.4254/wjh.v8.i13.591.

6. Zhu SL, Zhong JH, Ke Y, Ma L, You XM, Li LQ. Efficacy of hepatic resection vs. transarterial chemoembolization for solitary huge hepatocellular carcinoma. World J Gastroenterol 2015; 21(32):9630-7. doi: 10.3748/wjg. v21.i32.9630.

7. Ren Z, Yong XU, Zhu SJH. Indocyanine green retention test avoiding liver failure after hepatectomy for hepatolithiasis. Hepatogastroenterrol 2012; 59(115):782-4. doi: 10.5754/ hge11453.

8. Kim H, Ahn SW, Hong SK, Yoon KC, Kim HS, Choi YR, et al. Survival benefit of liver resection for barcelona clinic liver cancer stage B hepatocellular carcinoma. Burg J Surg 2017; 104(8):1045-52. doi: 10.1002/bjs.10541.

9. Fang Q, Xie QS, Chen JM, Shan SL, Xie K, Geng XP, et al. Long-term outcomes after hepatectomy of huge hepatocellular carcinoma: A single-center experience in China. Hepatobiliary Pancreat Dis Int 2019; 18(6):532-7. doi: 10.1016/j.hbpd.2019.09.001.

10. Wakayama K, Kamiyama T, Yokoo H, Orimo T, Shimada S, Einama $\mathrm{T}$, et al. Huge hepatocellular carcinoma greater than $10 \mathrm{~cm}$ in diameter worsens prognosis by causing distant recurrence after curative resection. J Surg Oncol 2017; 115(3):324-9. doi: 10.1002/jso.24501. 